\title{
A Robust Denoising Algorithm for Sounds of Musical Instruments Using Wavelet Packet Transform
}

\author{
Raghavendra Sharma, Vuppuluri Prem Pyara \\ Department of Electrical Engineering, Dayalbagh Educational Institute, Agra, India \\ Email: raghsharma2000@yahoo.com
}

Received September 17, 2013; revised October 17, 2013; accepted October 24, 2013

Copyright (c) 2013 Raghavendra Sharma, Vuppuluri Prem Pyara. This is an open access article distributed under the Creative Commons Attribution License, which permits unrestricted use, distribution, and reproduction in any medium, provided the original work is properly cited.

\begin{abstract}
In this paper, a robust DWPT based adaptive bock algorithm with modified threshold for denoising the sounds of musical instruments shehnai, dafli and flute is proposed. The signal is first segmented into multiple blocks depending upon the minimum mean square criteria in each block, and then thresholding methods are used for each block. All the blocks obtained after denoising the individual block are concatenated to get the final denoised signal. The discrete wavelet packet transform provides more coefficients than the conventional discrete wavelet transform (DWT), representing additional subtle detail of the signal but decision of optimal decomposition level is very important. When the sound signal corrupted with additive white Gaussian noise is passed through this algorithm, the obtained peak signal to noise ratio (PSNR) depends upon the level of decomposition along with shape of the wavelet. Hence, the optimal wavelet and level of decomposition may be different for each signal. The obtained denoised signal with this algorithm is close to the original signal.
\end{abstract}

Keywords: DWPT; Adaptive Block Denoising; Peak Signal to Noise Ratio; Wavelet Thresholding

\section{Introduction}

In the field of denoising the sounds of musical instruments, time frequency based transforms play an important role. They allow us to work with a sound signal from both time and frequency perspectives simultaneously. Such transforms have traditionally been useful in studying the nature of the sound signal, noise, and in facilitating the application of aesthetically interesting and novel modification to specific sound signals [1]. We are interested in a transform that is useful in working with musical instrument sound signals, and we look at the application of the discrete wavelet packet transform (DWPT) to remove the additive white Gaussian noise. There are several reasons for choosing the DWPT, it is inherently multi-resolution, making it more suited to human psychoacoustics than fixed resolution transforms as short time Fourier transform (STFT) [2]. It is easily reconfigured to allocate time frequency resolution in different ways through various basis selection approaches. Furthermore, efficient discrete time algorithms are available, and the transform basis function is inherently time localized without the introduction of a separate window function.
Signals may be transformed, modified and re-synthesized using DWPT without affecting the quality of the signal [3].

Noise has been a major problem for all signal processing applications. An unwanted signal gets superimposed over clean undisturbed signal. Noise exists in high frequency, but the sound signal is primarily low frequency. Since the wavelet transform decomposes the signal into approximation (low frequency) and detail (high frequency) coefficients [4,5], much of the noise is concentrated in detail coefficients. This suggests a method to denoise the signal, simply reducing the size of the detail coefficients before using them to reconstruct the signal, which is called thresholding or shrinkage rule [6]. We cannot eliminate the detail coefficients entirely, because they contain some important information of the signal. Various kinds of thresholding have been proposed in literature [7], but the choice depends upon the application at hand. The two important types of thresholing, hard and soft have been used to denoise the signal. In hard thresholding the wavelet coefficients below the given threshold are set to zero, but in soft thresholding the wavelet coefficients are reduced by a quantity equal to the threshold 
value. The extension of discrete wavelet transform is discrete wavelet packet transform in which we split both low pass and high pass filters at all scales in filter bank implementation to obtain flexible and detail analysis transform for denoising the sound signals [8]. In [9], wavelet packet approach which deals with heterogeneous noise for preprocessing of mass spectrometry data is discussed which incorporate a variance change point detection method in thresholding. Wavelet packet method has been used to reduce the Additive White Gaussian Noise from the speech signal which shows significant SNR improvement [10]. The rest of the article is organized as follows: In Section 2, brief theory of discrete wavelet packet transform (DWPT) is given. Wavelet packet adaptive block denoising scheme is discussed in Section 3, which is preceded by block denoising algorithm based on DWPT in Section 4. The various experimental results are discussed in Section 5. Section 6 gives the concluding remarks based on the experimental results.

\section{Discrete Wavelet Packet Transform (DWPT)}

Discrete wavelet packet transforms are used to get the advantage of better frequency resolution representation. When the wavelet transform is generalized to wavelet packet transform, not only the low pass filter output is decomposed through further filtering, but the high pass filter output decomposed as well. The ability to decompose the high pass filter outputs means that the wavelet packet allows for more than one basis function at a given scale, versus the wavelet transform which has one basis function at each scale other than the deepest level, where it has two.

The set of wavelet packets collectively make up the complete family of possible basis, and many potential basis can be constructed from them. If only the low pass filter is decomposed, the result is wavelet basis. If all low pass and high pass filters are decomposed, the complete tree basis results. This basis has the time frequency partitioning like STFT. Between these two extremes lie a large number of possible basis and their associated sub trees. Nodes can be merged or split based on the requirement of application. In all cases, the leaves of each connected sub tree of the complete wavelet packet tree from the basis of initial space; they span the space in linearly independent fashion. The tree diagram of a depth-3 complete tree basis is shown in the Figure 1.

As with the wavelet transform tree diagram in [11], denotes the depth within the transform and $k$ the position of each node $(j, k)$, but now the position index conveys more information, specifically which wavelet packet it corresponds to a given scale. We refer to the associate wavelet packet as $w_{j, k, p}$ analogus to the

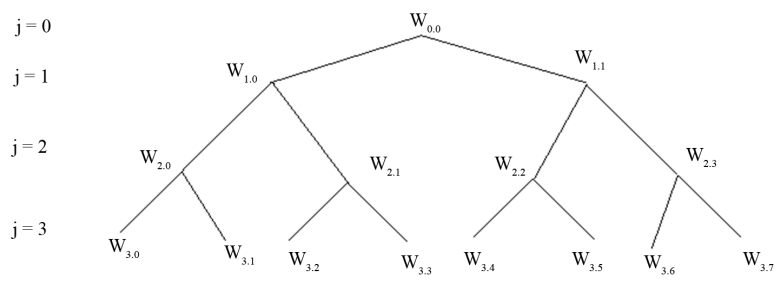

Figure 1. Depth-3 discrete wavelet packet transform tree.

wavelet $w_{k, p}$. The tree diagram does not convey time domain information, so the index $p$ is not used in node naming. Hence in wavelet packet, if all the packets are at the same scale, we may simply refer to them as $w_{k}$ as shown in the Figure 1.

Furthermore, $w_{j, k}$ is either the scaling function, or derived from the scaling function. DWPT does not require the explicit definition of wavelet, only filter definitions are enough. To see the wavelet packet at given level of decomposition, we can do a recursion of them at each node moving down the tree, to get the wavelet at next level. Specifically, if we split a wavelet packet node at level $j$ and position $k$ into two nodes at level $j+1$ and locations $2 k$ and $2 k+1$, we get the following two packets:

$$
w_{j+1,2 k}(n)=\sum_{m=0}^{M-1} h_{0}[m] w_{j, k}(2 n-m)
$$

and

$$
w_{j+1,2 k+1}(n)=\sum_{m=0}^{M-1} h_{1}[m] w_{j, k}(2 n-m)
$$

Then the wavelet packet transform coefficients $c_{j, k, p}$ are given by:

$$
c_{j, k, p}=\sum_{m=0}^{M-1} s[m] w_{j, k, p}(m)
$$

And the original signal can be expressed in terms of these coefficients and the corresponding wavelet packets as:

$$
s[m]=\sum_{j, k, p} c_{j, k, p} w_{j, k, p}[m],
$$

$(j, k) \in$ all leaf nodes of basis.

where $\mathrm{p}$ ranges over all time offsets at scale $\mathrm{j}$ for which signal $\mathrm{s}$ is defined.

\section{Wavelet Packet Adaptive Block Denoising}

The wavelet packet based denoising technique employs the decomposition concept in adaptive base of wavelets. This technique is efficient in denoising the musical sound signal corrupted with additive white Gaussian noise (AWGN), which is evenly distributed over the entire signal, and removal of AWGN from noisy signal is difficult task. Donoho and Johnstone pioneered the work of 
filtering the additive white Gaussian noise using wavelet thresholding [12]. The block denoising is explained in the following sub sections:

\subsection{Thresholding Based Denoising}

A noise reduction technique developed by donoho, uses the wavelet coefficients contraction and its principle consists of three steps;

1) Apply discrete wavelet transform to noisy signal:

$$
W \cdot y=W \cdot s+W \cdot z
$$

where $y, s, z$ and $W$ are the noisy musical instrument sound, original clean sound signal, noise signal and the matrix associated to the discrete wavelet transform respectively.

2) Threshold the obtained wavelet coefficients.

3) Reconstruct the desired signal by applying the inverse wavelet transform to the thresholded wavelet coefficients.

The thresholding function which is also known as wavelet shrinkage function is categorized as hard thresholding and soft thresholding function. The hard thresholdingfunction retains the wavelet coefficients which are greater than the threshold $\lambda$ and sets all other to zero. The hard thresholding is defined as:

$$
f_{h}(x)= \begin{cases}x, & \text { if }|x| \geq \lambda \\ 0, & \text { otherwise }\end{cases}
$$

The threshold $\lambda$ is chosen according to the signal energy and the standard deviation $\sigma$ of the noise. If the wavelet coefficient is greater than $\lambda$, then it is assumed that it is significant and contributes to the original signal. Otherwise it is due to the noise and discarded. The soft thresholding function shrinks the wavelet coefficients by $\lambda$ towards zero. Hence this function is also called as shrinkage function. The soft thresholding function is defined as:

$$
f_{s}(x)= \begin{cases}x-\lambda, & \text { if }|x| \geq \lambda \\ 0, & \text { if }|x|<\lambda \\ x+\lambda, & \text { if }|x| \leq \lambda\end{cases}
$$

In [13], we see that the soft thresholding gives lesser mean square error. Due to this reason soft thresholding is preferred over hard thresholding, but in case of some signals, we could see that hard thresholding results in lesser amount of mean square error.

\subsection{Block Selection}

Most of the musical instrument sound signals are far too long to be processed in their entirety; for example a 10 second sarangi sound signal sampled at $44.1 \mathrm{KHz}$ will contain 441,000 samples. Thus, as with spectral methods of noise reduction, it is necessary to divide the time domain signal in multiple blocks and process the each block individually. The block formation of the signal is shown in the Figure 2. The important task is to choose the block length. Berger et al. [14] shows that, blocks which are too shorts fail to pick important time structures of the signal. Conversely, blocks which are too long miss cause the algorithm to miss the important transient details in the musical instrument sound signal. Due to the binary splitting nature of the tree bases in wavelet analysis to decompose the signal, it is better to choose the length of each block with a number of samples to a power of two.

As discussed previously, the block size chosen must strike a balance between being able to pick up important transient detail in the sound signal, as well as recognizing longer duration, sustained events. Tables 1 and $\mathbf{2}$ shows the PSNR values which are quality measures, obtained for various block sizes and for different signals.

Tables 1 and 2 show that the PSNR values for different wavelets are varying with the block size. Hence the optimum block is that for which we have maximum

\begin{tabular}{|c|c|c|c|c|c|}
\hline Samples/block length (ms) & haar & $\mathrm{db} 10$ & sym3 & coif5 & dmey \\
\hline $1024 / 23$ & 23.06 & 33.99 & 30.99 & 36.42 & 36.41 \\
\hline $2048 / 46$ & 23.37 & 34.82 & 30.27 & 36.07 & 36.57 \\
\hline $4096 / 92$ & 23.96 & 36.45 & 31.73 & 36.9 & 39.62 \\
\hline $8192 / 185$ & 22.50 & 34.06 & 30.73 & 36.81 & 38.37 \\
\hline $16,384 / 371$ & 23.12 & 35.85 & 30.86 & 34.59 & 36.31 \\
\hline $32,768 / 743$ & 23.60 & 34.64 & 31.28 & 35.38 & 35.76 \\
\hline $65,536 / 1486$ & 22.50 & 33.21 & 30.95 & 35.25 & 36.25 \\
\hline
\end{tabular}
PSNR or minimum mean square error. The optimal block

Table 1. PSNR values obtained for different block length on shehnai sound with different wavelets. 
Table 2. PSNR values obtained for different block length on dafli sound with different wavelets.

\begin{tabular}{cccccc}
\hline Samples/block length (ms) & haar & db10 & sym3 & coif5 & dmey \\
\hline $1024 / 23$ & 07.39 & 13.20 & 09.22 & 08.91 & 34.15 \\
$2048 / 46$ & 32.74 & 34.84 & 23.43 & 37.66 & 37.22 \\
$4096 / 92$ & 35.78 & 35.42 & 36.96 & 46.76 & 37.28 \\
$8192 / 185$ & 37.17 & 44.95 & 43.17 & 49.68 & 42.57 \\
$16,384 / 371$ & 43.77 & 50.17 & 45.63 & 42.58 & 47.20 \\
$32,768 / 743$ & 41.89 & 44.79 & 42.88 & 45.37 & 39.49 \\
$65,536 / 1486$ & 40.84 & 45.29 & 44.32 & & 38.54 \\
\hline
\end{tabular}

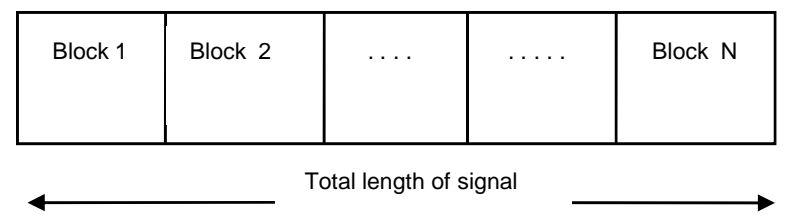

Figure 2. Block formation of signal

size for shehnai sound is 4096 samples and for dafli 16,384 samples. The informal listening test agree with this statement in a general sense, hence the block size is variable for musical instrument sound signals.

\subsection{Threshold Selection}

Donoho and Johnstone derived a general optimal universal threshold for the Gaussian white noise under a mean square error (MSE) criterion described in [12]. However this threshold is not ideal for musical instrument sound signals due to poor correlation between the MSE and subjective quality and the more realistic presence of correlated noise. Here we use a new time frequency dependent threshold estimation method. In this method first of all the standard deviation of the noise, $\sigma$ is calculated for each block. For given $\sigma$, we calculate the threshold for each block. Noise component removal by thresholding the wavelet coefficients is based on the observation that in musical instrument sound signal, energy is mostly concentrated in small number of wavelet dimensions. The coefficients of these dimensions are relatively very large compared to other dimensions or to any other signal like noise that has its energy spread over a large number of coefficients. Hence by setting smaller coefficients to be zero, we can optimally eliminate noise while preserving important information of the signal. In wavelet domain noise is characterized by smaller coefficients, while signal energy is concentrated in larger coefficients. This feature is useful for eliminating noise from signal by choosing the appropriate threshold. Generally the selected threshold is multiplied by the median value of the detail coefficients at some specified level which is called threshold processing.
At each level of decomposition, the standard deviation of the noisy signal is calculated. The standard deviation is calculated by Equation (8):

$$
\sigma_{j}=\frac{\operatorname{median}\left(\left|c_{j}\right|\right)}{0.6745}
$$

where $c_{j}$ are high frequency wavelet coefficients at $j^{\text {th }}$ level of decomposition, which are used to identify the noise components and $\sigma_{j}$ is Median Absolute Deviation (MAD) at this level. This standard deviation can be further used to set the threshold value based on the noise energy at that level. The modified threshold value [15] can be obtained by the equation (9):

$$
T_{h}=k \cdot \sigma_{j} \sqrt{2 \log \left(L_{j} \log _{2} L_{j}\right)}
$$

where $T_{h}$ is threshold value, $L_{j}$ is the length of each block of noisy signal and $k$ is the constant whose value is varying between $0-1$. For determining the optimum threshold, value of $\mathrm{k}$ should be estimated.

\section{Denoising Algorithm}

The proposed wavelet packet based block denoising algorithm for reduction of white Gaussian noise is explained in the following steps:

1) Take a musical instrument sound signal of suitable length.

2) Add White Gaussian Noise to the original signal depending upon the standard deviation $\sigma$.

3) Divide the noisy signal into blocks of different length depending upon the length of the signal in time domain, and the number of samples should be to a power of two.

4) Determine the optimal block size based on minimum mean square error criteria.

5) Compute the discrete wavelet packet transform (DWPT) of one block of the noisy signal at level 1.

6) Estimate the standard deviation of the noise using Equation (8) and determine the threshold value using Equation (9), then apply the different thresholding techniques for time and level dependent wavelet co- 
efficients using Equations (6) and (7).

7) Take inverse discrete wavelet packet transform (IDWPT) of the coefficients obtained through step 6 , which has reduced noise.

8) Calculate mean square error (MSE), peak signal to noise ratio (PSNR) for denoised signal.

9) Repeat steps 4 to step 7 for other level of decomposition 2 - 5.

10)Concatenate all the blocks of the denoised signals obtained through step 8 and do averaging operation for MSE and PSNR of the musical instrument sound signal.

The complete DWPT based denoising algorithm is shown graphically in Figure 3.

\section{Results and Discussions}

The denoising algorithm developed in the previous section is applied to the sound samples of the various Indian musical instruments sampled at $44.1 \mathrm{~K}$ samples per second. For experimental purpose the sounds of three musical instruments shehnai, dafli and flute are taken. For comparing the performance of the various wavelets for musical instrument sound signals, six wavelets haar, db10, sym3, coif5, dmey and bior 2.2 are taken. Besides observing the performance of the wavelets, the effect of decomposition is also discussed.

For comparing the performance and measurement of quality of denoising, the peak signal to noise ratio (PSNR) is determined between the original signal $S_{i}$ and the signal denoised $S_{d}$, by our algorithm.

$$
\text { PSNR }=10 \log _{10}\left(\frac{S_{\max }^{2}}{\mathrm{MSE}}\right)
$$

where $S_{\max }$ is the maximum value of the signal and is given by,

$$
S_{\max }=\max \left(\max \left(S_{i}\right), \max \left(S_{d}\right)\right)
$$

And MSE is mean square error, given by:

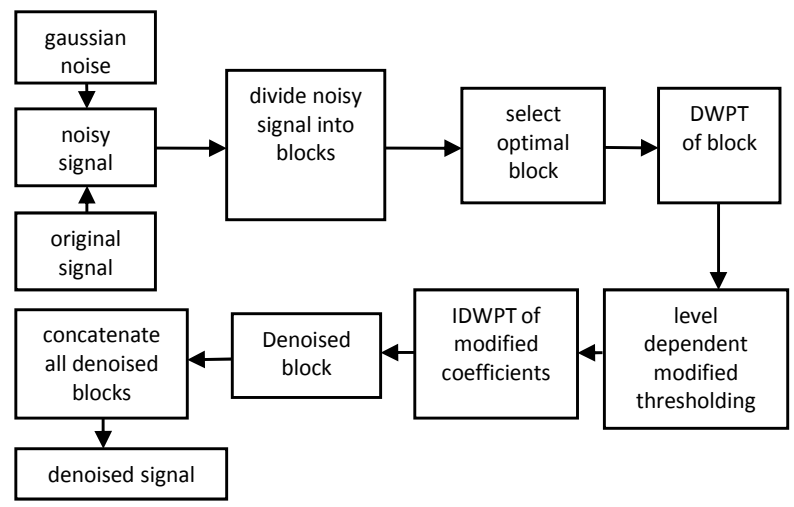

Figure 3. DWPT based block denoising algorithm with modified threshold.

$$
M S E=\frac{1}{N} \sum_{l=1}^{N}\left[S_{d}(l)-S_{i}(l)\right]^{2}
$$

where $\mathrm{N}$ is the length of the signal. The PSNR vaues obtained for different wavelets applied on shehnai, dafli and flute signals at different level of decomposition are shown in Tables 3-5. The additive white Gaussian noise is taken at $\sigma=0.1$, which is approximately $50 \%$ of the signal value.

It is observed from Tables 3-5 that the PSNR values are dependent upon the shape of the wavelet, type of thresholding and the level of decomposition. Hard thresholds are better than soft thresholds for denoising the musical instrument sound signals. The selection of level of decomposition plays a significant role, and should be optimal for best denoising results. Hence, the shehnai sound will give best results when denoised with db 10 wavelet at level 5, dafli sound with dmey at level 5 and flute sound with $\mathrm{db} 10$ at level 4 , respectively. The different signals denoised with optimal wavelet and level of decomposition are shown in the Figures 4-6.

\section{Conclusion}

Adaptive wavelet packet transform has been widely used in denoising the sounds of musical instruments and

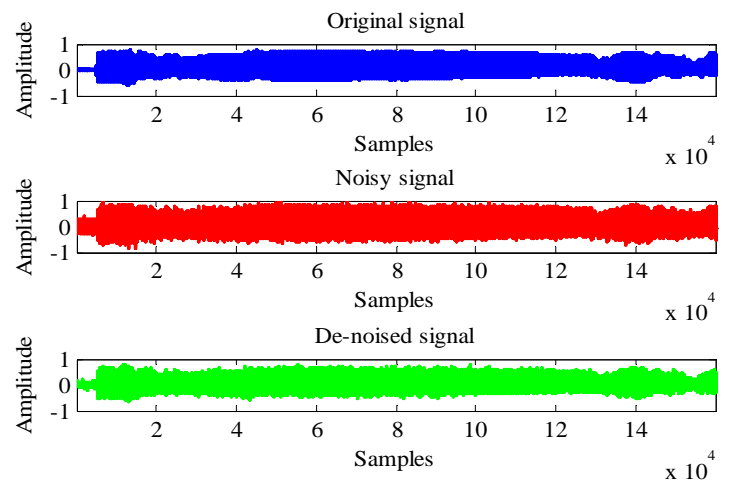

Figure 4. Original, noisy and denoised shehnai signal with db 10 at level 5 .

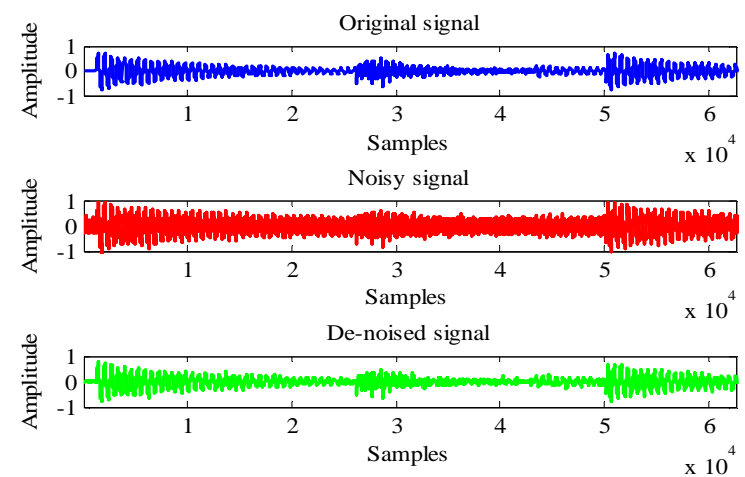

Figure 5. Original, noisy and denoised dafli signal with dmey at level 5. 
Table 3. PSNR values of shehnai sound after decompostion at different levels.

\begin{tabular}{|c|c|c|c|c|c|c|c|c|}
\hline \multirow{2}{*}{ Wavelet } & \multicolumn{2}{|c|}{ Level 2} & \multicolumn{2}{|c|}{ Level 3} & \multicolumn{2}{|c|}{ Level 4} & \multicolumn{2}{|c|}{ Level 5} \\
\hline & soft & hard & soft & hard & soft & hard & soft & hard \\
\hline haar & 26.02 & 31.33 & 18.57 & 18.72 & 23.09 & 27.65 & 16.89 & 25.32 \\
\hline db10 & 23.95 & 23.43 & 18.53 & 18.55 & 20.14 & 27.23 & 16.52 & 31.62 \\
\hline sym3 & 26.44 & 25.68 & 18.56 & 18.56 & 19.65 & 27.21 & 14.85 & 30.39 \\
\hline coif5 & 24.02 & 23.71 & 18.68 & 19.65 & 21.05 & 27.22 & 18.13 & 29.65 \\
\hline dmey & 23.92 & 23.78 & 18.57 & 18.76 & 23.34 & 23.48 & 22.76 & 23.43 \\
\hline bior2.2 & 31.06 & 26.27 & 18.44 & 18.54 & 25.26 & 25.75 & 20.61 & 26.93 \\
\hline
\end{tabular}

Table 4. PSNR values of dafli sound after decomposition at different levels.

\begin{tabular}{|c|c|c|c|c|c|c|c|c|}
\hline \multirow{2}{*}{ Wavelet } & \multicolumn{2}{|c|}{ Level 2} & \multicolumn{2}{|c|}{ Level 3} & \multicolumn{2}{|c|}{ Level 4} & \multicolumn{2}{|c|}{ Level 5} \\
\hline & soft & hard & soft & hard & soft & hard & soft & hard \\
\hline haar & 20.07 & 19.88 & 17.56 & 17.72 & 27.76 & 27.58 & 26.08 & 27.48 \\
\hline $\mathrm{db} 10$ & 20.14 & 19.56 & 17.66 & 17.75 & 24.30 & 24.56 & 26.05 & 27.67 \\
\hline sym3 & 20.12 & 19.86 & 17.46 & 17.56 & 26.12 & 25.24 & 26.92 & 26.93 \\
\hline coif5 & 19.65 & 20.05 & 17.50 & 17.59 & 25.88 & 24.88 & 27.05 & 26.79 \\
\hline dmey & 20.05 & 19.78 & 17.44 & 17.65 & 24.47 & 25.18 & 27.05 & 27.97 \\
\hline bior2.2 & 20.00 & 19.77 & 17.35 & 17.53 & 23.58 & 21.73 & 24.21 & 23.11 \\
\hline
\end{tabular}

Table 5. PSNR values of flute sound after decomposition at different levels.

\begin{tabular}{|c|c|c|c|c|c|c|c|c|}
\hline \multirow{2}{*}{ Wavelet } & \multicolumn{2}{|c|}{ Level 2} & \multicolumn{2}{|c|}{ Level 3} & \multicolumn{2}{|c|}{ Level 4} & \multicolumn{2}{|c|}{ Level 5} \\
\hline & soft & hard & soft & hard & soft & hard & soft & hard \\
\hline haar & 21.83 & 22.99 & 19.55 & 19.21 & 16.16 & 18.82 & 9.07 & 18.97 \\
\hline $\mathrm{db} 10$ & 23.73 & 24.02 & 19.09 & 19.40 & 13.65 & 35.71 & 11.31 & 33.00 \\
\hline sym3 & 24.50 & 24.64 & 19.36 & 19.52 & 16.49 & 28.64 & 11.48 & 30.47 \\
\hline coif5 & 23.98 & 24.10 & 19.40 & 19.63 & 13.75 & 33.98 & 11.42 & 29.68 \\
\hline dmey & 23.75 & 24.37 & 19.25 & 19.53 & 13.74 & 34.53 & 11.76 & 30.26 \\
\hline bior2.2 & 28.80 & 27.72 & 19.41 & 19.48 & 13.46 & 18.94 & 10.87 & 19.70 \\
\hline
\end{tabular}

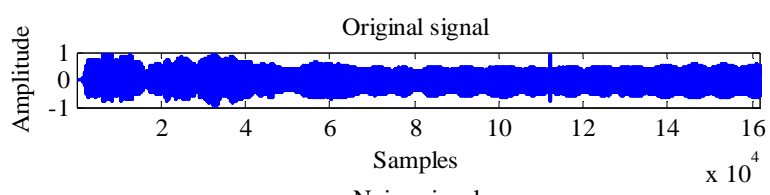

Noisy signal
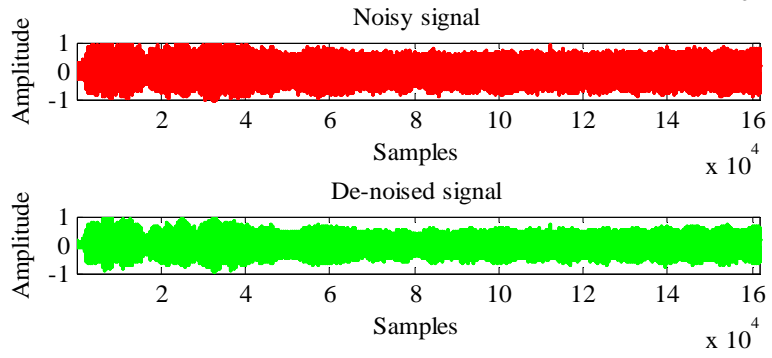

Figure 6. Original, noisy and denoised flute signal with db10 at level 4.
Providing better performance in terms of PSNR values than the other denoising techniques. In this paper, discrete wavelet packet transform is used for denoisingshehnai, dafli and flute sound signal corrupted with additive white Gaussian noise, 50\% of the signal strength. First, sound signal is divided into multiple blocks depending upon the optimal block size for each signal. Denoising of signal is performed with these optimal block sizes in wavelet packet domain by thresholding the wavelet coefficients at different level of decomposition. It is observed that hard thresholding gives better PSNR than soft thresholding at all the decomposition levels. The choice of the optimal level of decomposition is important, and different for each sound signal. If the level of decomposition is not optimal then the PSNR value 
will not be maximum, hence denoising will not be the best. Maximum PSNR value for shehnai sound is at level 5 with db10 wavelet, dafli at level 5 with dmey and flute at level 4 with db10 respectively. When each block is denoised, all the blocks are concatenated to form the final denoised signal. It is also observed that when modified threshold with is used, the PSNR values are increased. Higher thresholds remove the noise well but some parts of the original signal are also removed because it is not possible to remove the noise without affecting the original signal.

\section{REFERENCES}

[1] M. Lang, H. Guo, J. E. Odegard, C. S. Burrus and R. O. Wells, "Noise Reduction Using an Undecimated Discrete Wavelet Transform," IEEE Signal Processing Letters, Vol. 3, No. 1, 1996, pp. 10-12.

[2] J. Yang, Y. Wang, W. Xu and Q. Dai, "Image and Video Denoising Using Adaptive Dual Tree Discrete Wavelet Packets," IEEE Transaction on Circuit and Systems for Video Technology, Vol. 19, No. 5, 2009, pp. 642-655.

[3] B. J. Shankar and K. Duariswamy, "Wavelet Based Block Matching Process: An efficient Audio Denoising Technique," European Journal of Scientific Research, Vol. 48, No. 1, 2010, p. 16.

[4] R. Sharma and V. P. Pyara, "A Novel Approach to Synthesize Sounds of Some Indian Musical Instruments Using DWT," International Journal of Computer Applications, Vol. 45, No. 13, 2012, pp. 19-22.

[5] R. Sharma and V. P. Pyara, "A Comparative Analysis of Mean Square Error Adaptive Filter Algorithms for Generation of Modified Scaling and Wavelet Function," International Journal of Engineering Science and Technology, Vol. 4, No. 4, 2012, pp. 1396-1401.

[6] J. Yu and D. C. Liu, "Thresholding Based Wavelet Packet Methods for Doppler Ultrasound Signal Denoising,” IF-
MBE Proceedings Springer Verlag Berlin Heidelberg, Vol. 19, No. 9, 2008, pp. 408-412.

[7] T. Mourad, S. Lotfi and C. Adnen, "Spectral Entropy Employment in Speech Enhancement Based on Wavelet Packet," International Journal of Computer and Information Engineering, Vol. 1, No. 7, 2007, pp. 404-411.

[8] N. S. Nehe and R. S. Holambe, "DWT and LPC Based Feature Extraction Methods for Isolated Word Recognition,” EURASIP Journal of Audio, Speech and Music Processing, Vol. 7, No. 1, 2012, pp. 1-7.\# http://dx.doi.org/10.1186/1687-4722-2012-7

[9] D. Kwon, M. Vannucci, J. J. Song, J. Jeong and R. M. Pfeiffer, "A Novel Wavelet Based Thresholding Method for the Pre-Processing of Mass Spectrometry Data That Accounts for Heterogeneous Noise,” Proteomics, Vol. 8, No. 15, 2008, pp. 3019-3029.

[10] Y. Ren, M. T. Johnson and J. Tao, "Perceptually Motivated Wavelet Packet Transform for Bio-Acoustic Signal Enhancement," Journal of Acoustic Society of America, Vol. 124, No. 1, 2008, pp. 316-327.

[11] K. Ramchandran and M. Vetterli, "Best Wavelet Packet Bases in a Rate-distortion Sense," IEEE Transaction on Image Processing, Vol. 2, No. 2, 1993, pp. 160-175.

[12] D. L. Donoho and I. M. Johnstone, "Adapting to Unknown Smoothness via Wavelet Shrinkage," Journal of the American Statistical Association, Vol. 90, No. 432, 1995, pp. 1200-1224.

[13] S. G. Chang, B. Yu and M. Vetterli, "Adaptive Wavelet Thresholding for Image Denoising and Compression," IEEE Transaction on Image Processing, Vol. 9, No. 9, 2000, pp. 1532-1546.

[14] J. Berger, R. R. Coifman and J. G Maxim, "Removing Noise from Music Using Local Trigonometric Bases and Wavelet Packets," Journal of The Audio Engineering Society, Vol. 42, No. 10, 1994, pp. 808-818.

[15] M. T. Johnson, X. Yuan and Y. Ren, "Speech Signal Enhancement through Adaptive Wavelet Thresholding," Speech Communication, Vol. 49, No. 2, 2007, pp. 123-133. 